\title{
Osteoimmune Modulation and Guided Osteogenesis Promoted by Barrier Membranes Incorporated with S-Nitrosoglutathione (GSNO) and Mesenchymal Stem Cell-Derived Exosomes
}

This article was published in the following Dove Press journal:

International Journal of Nanomedicine

Xin Wang, ${ }^{1,2, *}$ Jun Ao, ${ }^{1, *}$ Haiping Lu,' Qingyu Zhao,' Yaping Ma, ',2 Jun Zhang,' Hao Ren, ${ }^{3}$ Yi Zhang ${ }^{2,4}$

'Department of Orthopaedic Surgery, Affiliated Hospital of Zunyi Medical University, Zunyi 563000, Guizhou, People's Republic of China; ${ }^{2}$ Joint Orthopaedic Research Center of Zunyi Medical University \& University of Rochester Medical Center (JCMR-ZMU \& URMC), Zunyi Medical University, Zunyi 563000, Guizhou, People's Republic of China; ${ }^{3}$ Shenzhen Institute for Innovation and Translational Medicine, Shenzhen International Biological Valley-Life Science Industrial Park, Shenzhen, Guangdong 5 I8II9, People's Republic of China; ${ }^{4}$ Department of Hygiene Toxicology, School of Public Health, Zunyi Medical University, Zunyi 563000, Guizhou, People's Republic of China

*These authors contributed equally to this work

Correspondence: Hao Ren

Shenzhen Institute for Innovation and Translational MedicineShenzhen International Biological Valley-Life Science Industrial Park, Shenzhen 518II9,

Guangdong, People's Republic of China

Tel +86 I73 69724036

Fax +86 755-8420 0009

Email renhao0421@foxmail.com

Yi Zhang

Joint Orthopaedic Research Center of Zunyi

Medical University \& University of

Rochester Medical Center (JCMR-ZMU \&

URMC), Zunyi Medical University, Zunyi

563000 , Guizhou, People's Republic of China

Tel +86 I827566 2009

Fax $+86851-2860955$ I

Email zhangyizmr@I63.com
Background: The use of polycaprolactone (PCL) for bone defects in a clinical setting is limited due to a lack of bioactivity. Exosomes derived from mesenchymal stem cells (MSCs) have an important immunoregulatory potential and together with S-nitrosoglutathione (GSNO) they possess therapeutic potential for bone regeneration.

Materials and Methods: In this study, PCL was modified with GSNO and MSC-derived exosomes and the impact on macrophages and osteogenes is evaluated.

Results: MSC-derived exosomes exhibited a cup-shaped morphology and were internalized by macrophages and human bone marrow-derived mesenchymal stromal cells (hBMSCs). The pattern of internalization of scaffold-immobilized exosomes was similar in RAW264.7 cells and hBMSCs after $4 \mathrm{~h}$ and $24 \mathrm{~h}$ of co-culture. Assessment of macrophage morphology under inflammatory conditions by scanning electronic microscopy (SEM) and confocal microscopy demonstrated macrophages were significantly elongated and expression of proinflammatory genes markedly decreased when co-cultured with PCL/PDA + GSNO + exosome scaffolds. Furthermore, this scaffold modification significantly enhanced osteogenic differentiation of hBMSCs.

Discussion: This study demonstrated the possibility of using a GSNO- and exosome-based strategy to adapt barrier membrane scaffolds. PCL/PDA + GSNO + exosome scaffolds may serve as an important barrier membrane for osteogenesis and tissue regeneration.

Keywords: polycaprolactone, mesenchymal stem cells, exosomes, S-nitrosoglutathione, osteogenesis

\section{Introduction}

Large bone defects are characterized by delayed bone healing or non-healing and account for $2.5 \%$ and $10 \%$ respectively of total bone fracture cases ${ }^{1}$. At present, it represents one of the most common health problems worldwide. From the perspective of clinical management, the treatment of large bone defects is a critical issue for orthopedic surgeons. ${ }^{2}$ The ideal therapeutic strategy for restoring large bone defects is to rebuild the damaged bone using autologous cancellous bone grafting from the iliac crest of the patient however autologous bone graft materials are not always available and this can lead to additional pain and donor site morbidity. ${ }^{3}$ In addition, despite conventional treatment for controlling local infection and inflammation of large bone defects by surgical fixation, tissue regeneration remains 
inadequate and this requires the development of novel therapeutic intervention solutions. ${ }^{4,5}$

Recent advances in tissue engineering have facilitated development of various regenerative treatment modalities aimed at achieving controlled tissue regeneration. ${ }^{6}$ For instance, a variety of cell-based therapeutic strategies, including adipose tissue-derived stem cells (ADSCs) ${ }^{6,7}$ and bone marrow mesenchymal stromal cells (BM-MSCs) ${ }^{8}$ have been found to promote bone regeneration while other cytotherapeutic regenerative methods, such as cell sheetguided tissue regeneration or bioactive factors alone and in combination have been assessed in several clinical trials. ${ }^{9}$ However, the labor- and time-intensive nature of cytotherapeutic regenerative methods such as cell culture and cell sheet fabrication limits the widespread use of this technology. ${ }^{10}$ Exosomes are membrane vesicles which are naturally secreted from most cells in culture ${ }^{11}$ and mediate intercellular communication by delivering bioactive molecules such as proteins and nucleic acids to recipient cells. ${ }^{12}$ Recently, a regulatory role has been attributed to mesenchymal stem cells (MSCs)-derived exosomes in various physiological and pathological contexts, indicating the possible therapeutic potential of MSC-derived exosomes. The therapeutic effects of MSC-derived exosomes have been studied in vivo in a number of diseases models, such as myocardial infarction, ${ }^{13}$ liver disease, ${ }^{14}$ kidney injury, ${ }^{15}$ and lung injury. ${ }^{16}$ Through the delivery of their endogenous cargo including miRNAs and proteins, MSC-derived exosomes exert anti-inflammatory effects and promote tissue regeneration by enhancing immune signaling, cell proliferation and differentiation, and angiogenesis. ${ }^{17}$ S-nitrosoglutathione (GSNO), a s-nitrosated derivative of glutathione and has been shown to modulate fibrin structure and limit platelet activation resulting in a decrease in coagulation and thrombosis. ${ }^{18}$ A rat periodontitis model demonstrated local intragingival injections of GSNO significantly reduced inflammation and decreased alveolar bone destruction. ${ }^{19}$ Our results from a previous study further corroborate the regulatory properties of GSNO on blood clotting architecture which in turn, may play a pivotal role in early bone regeneration. ${ }^{20}$ Importantly, GSNO has also been clinically tested as an anti-platelet agent in respiratory disease, myocardial infarction, and antimicrobial therapy. ${ }^{21}$

Polycaprolactone (PCL) is a bioresorbable and biocompatible polymer, which has been approved by the US Food and Drug Administration (FDA) for clinical use in bone and cartilage repair. ${ }^{22}$ However, its lack of bioactivity limits its application for bone tissue engineering. ${ }^{23}$ In this study, we generated a hybrid PCL scaffold consisting of GSNO and/or MSC-derived exosomes to investigate if these modifications would promote osteogenesis and bone regeneration. Our results suggest that altering the osteogenic environment using bioactive agents such as GSNO and MSC-derived exosomes is a valid prospective therapeutic approach to augment osteogenesis.

\section{Materials and Methods \\ Reagents}

Total Exosome Isolation Reagent (from cell culture media) was purchased from Thermo Fisher Scientific (catalog number 4478359; China). PKH67 Green Fluorescent Cell Linker Kit for General Cell Membrane Labeling (catalog number MINI67-1KT, China) and lipopolysaccharide (LPS, catalog number L4391, Escherichia coli 0111: B4) were purchased from Sigma-Aldrich (China). Anti-alkaline phosphatase (ALP) rabbit antibody (ab108337) and fluorescein isothiocyanate-conjugated goat anti-rabbit secondary IgG antibody (ab6717) were acquired from Abcam (China).

\section{Scaffold Fabrication and Modification}

PCL scaffolds were prepared by electrospinning. In brief, $10 \%(\mathrm{w} / \mathrm{v})$ PCL solution was prepared by dissolving PCL in 1,1,1,3,3,3-hexafluoro-2-propanol (HFP) (Sigma-Aldrich). The solution was loaded into a syringe and fed through a syringe pump at a flow rate of $2 \mathrm{~mL} / \mathrm{h}$. The needle was connected to a voltage of $20 \mathrm{kV}$ at a distance of $20 \mathrm{~cm}$. The PCL scaffolds were dried at room temperature by vacuum suction to evaporate residue. The PCL scaffolds were coated with Poly(dopamine) (PDA) coating to modify the surface. PCL scaffolds were immersed in a solution of dopamine hydrochloride $(4 \mathrm{mg} / \mathrm{mL})$ in $10 \mathrm{mM} \mathrm{pH}=8.5$ Tris-HCL buffer for $1 \mathrm{~h}$ with stirring before rinsing with Milli-Q water after which they are considered as PCL/PDA scaffolds. The PCL/PDA scaffolds were incubated with GSNO $(100 \mu \mathrm{M})$ overnight to obtain PCL/PDA + GSNO scaffolds. For exosome coating, exosomes (10 $\mu \mathrm{g}$ protein equivalent) were incubated with either PCL/PDA or PCL/PDA + GSNO scaffolds for $1 \mathrm{~h}$ at room temperature and then rinsed with sterile phosphate-buffered saline (PBS).

\section{Cell Culture}

Murine macrophage-like cell line, RAW 264.7 (ATCC $^{\circledR}$ TIB-71 $\left.{ }^{\mathrm{TM}}\right)$ were cultured in Dulbecco's Modified Eagle's Medium (DMEM; Life Technologies Pty Ltd., China) supplemented with $10 \%$ of heat-inactivated fetal bovine 
serum (FBS; Biological Industries, LTD, Beit Haemek, Israel), and $1 \%(\mathrm{v} / \mathrm{v})$ penicillin/streptomycin (Solarbio, Beijing, China) in a humidified incubator at $37^{\circ} \mathrm{C}$ and $5 \%$ $\mathrm{CO}_{2}$. Human bone marrow-derived mesenchymal stromal cells (hBMSCs) were isolated from bone marrow samples of patients undergoing surgery at Zunyi Medical University with written informed consent. The handling of human tissue samples was approved by the Zunyi Medical University Ethics Committee (Ethic approval number: Zun Yi Lun Shen 2017-1-048) and this study was conducted in accordance with the Declaration of Helsinki. hBMSCs were maintained in DMEM containing $10 \%$ of FBS and supplemented with $1 \%(\mathrm{v} / \mathrm{v})$ penicillin/ streptomycin. After rinsing trice with PBS, conditioned medium was collected after culturing with serum-free DMEM. The conditioned medium was stored at $-80^{\circ} \mathrm{C}$ until the exosome isolation.

\section{Isolation of MSC-Derived Exosomes}

Exosomes were isolated using Total Exosome Isolation Reagent (from cell culture media) according to the manufacturer's protocol. Briefly, cell debris was removed from the conditioned media by centrifugation at $2000 \times \mathrm{g}$ for 30 minutes and the supernatant transferred to a new tube. Onehalf volume of the Total Exosome Isolation Reagent (from cell culture media) was added to the supernatant, vortexed and incubated at $4{ }^{\circ} \mathrm{C}$ overnight. The following day the isolated exosomes were centrifuged at $10,000 \times g$ for $1 \mathrm{~h}$ at $4^{\circ} \mathrm{C}$ and the supernatant discarded. Exosomes were resuspended in $50 \mu \mathrm{L}$ PBS, aliquoted and stored at $-80^{\circ} \mathrm{C}$.

\section{Transmission Electron Microscopy (TEM)}

To visualize isolated exosomes by TEM, carbon/formvarcoated $\mathrm{Cu}$ TEM grids were placed on $5 \mu \mathrm{L}$ exosome samples for $10 \mathrm{~min}$. The grids were stained with $1 \%$ uranyl acetate (UA) for $10 \mathrm{~s}$ before washing twice with deionized water for $20 \mathrm{~s}$. The grids were dried using Whatman filter paper. TEM images were taken with a TEM (JEM-1400, JOEL, Japan) at $80 \mathrm{kV}$.

\section{Nanoparticle Tracking Analysis (NTA)}

NTA was performed using a Malvern NanoSight NS300 (Malvern Instruments, United Kingdom). The samples (diluted 1:500 in PBS) were loaded into $1 \mathrm{~mL}$ sterile syringes (BD Discardit II, New Jersey, USA) and infused into the sample chamber at a flow rate of $20 \mu \mathrm{L} / \mathrm{s}$. All infusions were performed at room temperature. 5 videos of
$60 \mathrm{~s}$ were taken, and particle sizes were analyzed by NTA software (Malvern Instruments, United Kingdom).

\section{Scanning Electron Microscopy (SEM)}

Specimens were rinsed twice with PBS, and fixed in 3\% glutaraldehyde in $0.1 \mathrm{M}$ sodium cacodylate buffer overnight. After rinsing 3 times with $0.1 \mathrm{M}$ sodium cacodylate buffer (10 $\mathrm{min}$ each), the scaffolds were post-fixed with $4 \%$ osmium, and ethanol gradient dehydration. Specimens were mounted on carbon tabs, and sputter coated with gold-palladium. All specimens were analyzed using a Zeiss SEM (Carl Zeiss NTS, Germany).

\section{PKH67 Labeling of Exosomes in vitro}

Purified exosomes were labeled with the green fluorescent membrane marker, PKH67 according to the manufacturer's instructions. Briefly, exosomes were suspended in $1 \mathrm{~mL}$ of diluent $\mathrm{C}$ to which $4 \mu \mathrm{L}$ of PKH67 was added and incubated for $5 \mathrm{~min}$. The labeling process was stopped by adding an equal volume of DMEM with $1 \%$ exosome-free FBS and incubating for a further $1 \mathrm{~min}$. The mix was then incubated with $1 \mathrm{~mL}$ of DMEM containing $10 \%$ of exosome-free FBS to which RAW 264.7 cells and hBMSCs were added and incubated at $37^{\circ} \mathrm{C}$ for $12 \mathrm{~h}, 24 \mathrm{~h}$, and $48 \mathrm{~h}$. Cellular uptake and internalization of exosomes were captured using an inverted confocal microscope with a $40 \times 1.3$ NA oil objective (Leica DM IRB, Leica, Wetzlar, Germany).

\section{XTT Assay}

To evaluate cell proliferation, the XTT assay kit (Abcam; ab232856) was used according to the manufacturer's instructions. Absorbance was read on a microplate reader (Benchmark Plus, USA) at $450 \mathrm{~nm}$.

\section{RNA Extraction, cDNA Synthesis, and Real-Time Quantitative Polymerase Chain Reaction (RT-PCR)}

RAW 264.7 cells were seeded at a density of $2 \times 10^{5} /$ well and stimulated with $1 \mu \mathrm{g} / \mathrm{mL}$ LPS for $12 \mathrm{~h}$. Following stimulation, the LPS was removed and the cells were washed with PBS. RAW 264.7 cells were then co-cultured with either PCL/PDA or PCL/PDA + GSNO scaffolds for a further $24 \mathrm{~h}$. Total RNA was isolated using Trizol reagent (Catalog number 15596026, Thermo Fisher Scientific) and RNA concentration measured using the NanoDrop 8000 spectrophotometer (NanoDrop Technologies Inc., USA). cDNA was synthesized using the RevertAid First Strand cDNA Synthesis Kit (Catalog number 
K1622, Thermo Fisher Scientific) according to manufacturer's instruction and RT-PCR performed using SYBR Green qPCR Master Mix (Life Technologies Pty Ltd., China) on an ABI Prism 7500 Thermal Cycler (Applied Biosystems, USA). All primer sequences (Supplementary Table 1) were analyzed for target specificity using Primer BLAST and purchased from Sigma-Aldrich. Expression of mRNA was normalized to the housekeeping gene glyceraldehyde 3-phosphate dehydrogenase (GAPDH). The difference between the mean $\mathrm{Ct}$ values of the gene of interest and GAPDH was designated $\Delta \mathrm{Ct}$ and the relative expression was calculated using the comparative $\mathrm{Ct}\left(2^{-\Delta \Delta \mathrm{CT}}\right)$ method. $^{24}$

\section{Determining ALP Activity in hBMSCs in Response to Different Scaffolds}

The expression of ALP was detected by immunofluorescence staining. Briefly, hBMSCs at a density of $2 \times 10^{5} /$ well were co-cultured in osteogenic medium (10\% DMEM supplemented with $2 \mathrm{mM} \beta$-glycerophosphate, $100 \mu \mathrm{M}$ 1-ascorbic acid 2-phosphate, and $10 \mathrm{nM}$ dexamethasone; Sigma-Aldrich) with different scaffolds for $7 \mathrm{~d}$. Samples were fixed with $4 \%$ paraformaldehyde for $20 \mathrm{~min}$ at room temperature and cells were permeabilized with Triton X-100 for 5 min, blocked with $4 \%$ bovine serum albumin for $1 \mathrm{~h}$ at room temperature, and incubated with primary antibody for ALP overnight at $4{ }^{\circ} \mathrm{C}$. The following day, cells were washed 3 times with PBS and incubated with fluorescein isothiocyanate-conjugated goat anti-rabbit IgG secondary antibody. Actin was stained using Alexa Fluor 594-labeled phalloidin (catalog number A12381, Life Technologies Pty Ltd). Samples were prepared for inspection using an inverted confocal microscope with a $40 \times 1.3 \mathrm{NA}$ oil objective (Leica DM IRB, Leica, Wetzlar, Germany). ALP activity was determined using the Alkaline Phosphatase Assay Kit (ab83369, Abcam) according to the manufacturer's instructions. hBMSCs were co-cultured in osteogenic medium with either different scaffolds and ALP activity measured after $7 \mathrm{~d}$ of culture. Osteogenic gene expression was determined in hBMSCs co-cultured with different scaffolds in osteogenic medium and cells collected after $3 \mathrm{~d}$. RT-PCR was performed as described above.

\section{Statistical Analysis}

Results were represented as the mean \pm standard deviation $(\mathrm{SD}, \mathrm{n}=3)$. Statistical analysis was performed using GraphPad Prism 8.0 for Windows (GraphPad Software Inc., USA). For statistical evaluation, Student's $t$-test was used to assess statistically significant differences between two datasets and one way analysis of variance (One Way ANOVA) between three datasets. Statistical significance was accepted when the difference between datasets were $p<0.05$.

\section{Results}

\section{Characterization of Isolated Exosomes}

To characterize features of MSC-derived exosomes, we first assessed morphology by TEM. Exosomes isolated in this study (Figure 1A) exhibited a cup-shaped morphology which was consistent with previous published result. ${ }^{25}$ We next carried out nanoparticle tracking analysis (NTA) to characterize the concentration and size distribution of the isolated exosomes (Figure 1B). Figure 1B demonstrates size distribution peaks of approximately $50 \mathrm{~nm}$ and $120 \mathrm{~nm}$. To further validate the presence of exosomes, the green fluorescent membrane marker, PKH67 was utilized. MSC-derived exosomes were labeled with PKH67 and incubated with RAW 264.7 cells or hBMSCs. At the indicated time points, cells were collected and stained for confocal microscopy analysis to capture internalization of exosomes. In RAW 264.7 cells, fluorescence intensity increased at $48 \mathrm{~h}$ compared with at $12 \mathrm{~h}$ and $24 \mathrm{~h}$ time points, while in hBMSCs, exosome internalization exhibited a peri-nuclear pattern throughout the whole time course (Figure 1C and D). Collectively, these results suggest exosomes from MSCs were successfully isolated in our study.

\section{Characterization of Exosome Modified Surfaces}

We next evaluated whether MSC-derived exosomes could be immobilized onto different scaffolds. Exosome protein concentration was evaluated (Supplementary Figure 1), Exosomes labelled with PKH67 were immobilized onto PCL/PDA or PCL/PDA + GSNO scaffolds. As a control, PCL/PDA and PCL/PDA + GSNO scaffolds were incubated with PKH67 in the absence of exosomes. Confocal microscopy demonstrated green fluorescently labeled exosomes could be immobilized onto the surface of PCL/PDA and PCL/PDA + GSNO scaffolds (Figure 2C and D). Control PCL/PDA and PCL/PDA + GSNO scaffolds stained with PKH67 exhibited green fibers indicating immobilization of exosomes to scaffolds was specific and robust (Figure 2A and B).

\section{Internalization of Scaffold-Immobilized Exosomes by RAW264.7 Cells and hBMSCs}

To evaluate whether the scaffolds exhibited biotoxicity, we assessed hBMSC proliferation using the XTT assay. No significant differences in cell proliferation was observed between 

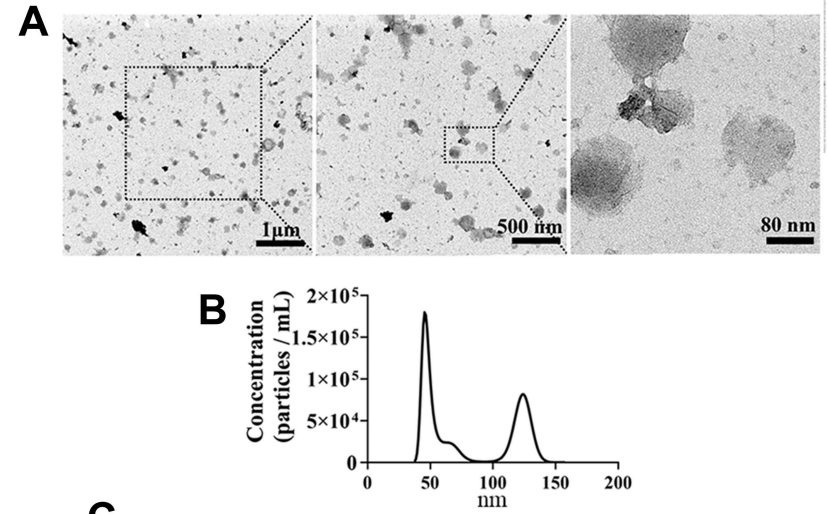

C

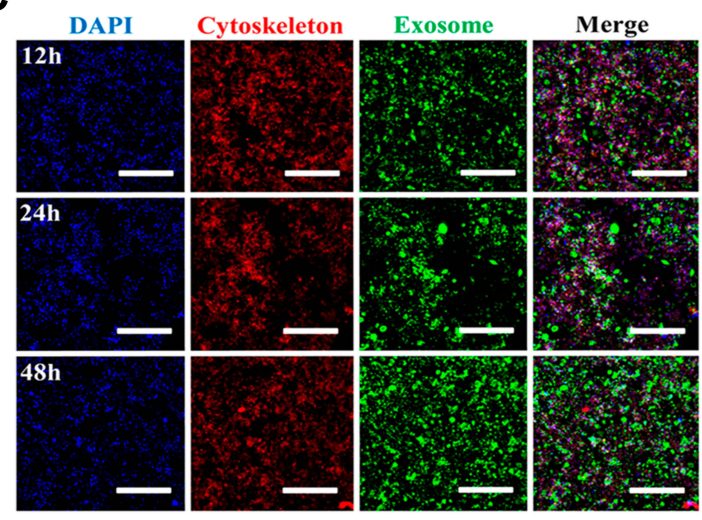

D

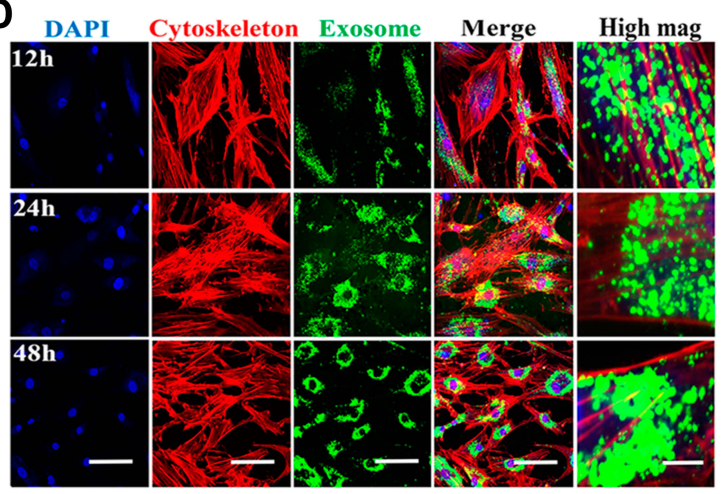

Figure I Isolation, characterization, and internalization of human bone marrowderived mesenchymal stromal cells (hBMSCs)-derived exosomes. (A) Morphology of exosomes under TEM. Negative staining demonstrated typical cup-shaped exosomal morphology. (B) Size distribution determined by NTA. (C) Representative confocal microscopy images of exosome internalization by RAW 264.7 cells at $12 \mathrm{~h}$, $24 \mathrm{~h}$, and 48h. Scale bars: $200 \mu \mathrm{m}$. (D) Representative confocal microscopy images of exosome internalization by hBMSCs at $12 \mathrm{~h}, 24 \mathrm{~h}$, and $48 \mathrm{~h}$. Scale bars: $100 \mu \mathrm{m}$ for low magnification images and $10 \mu \mathrm{m}$ for high magnification images. Results are representative of three independent experiments.

scaffold groups (PCL/PDA vs PCL/PDA + GNSO with or without immobilized exosomes; Supplementary Figure 2). To monitor exosome internalization by RAW 264.7 cells and hBMSCs, exosomes were labeled with PKH67, immobilized onto PCL/PDA and PCL/PDA + GSNO scaffolds and cocultured with RAW264.7 cells or hBMSCs (PCL/PDA +
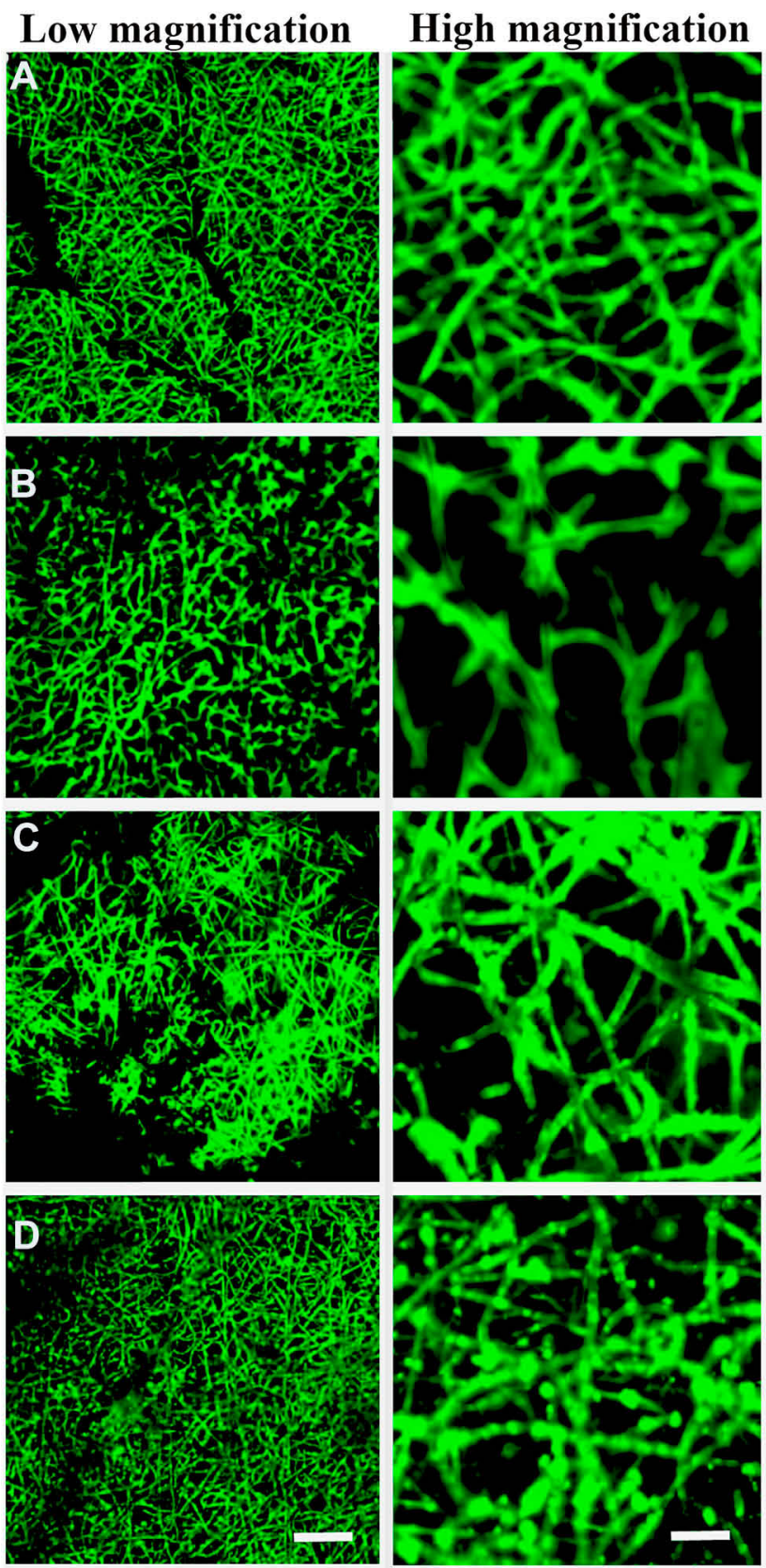

Figure 2 Surface characterization of exosomes immobilized onto PCL/PDA and PCL/ PDA + GNSO scaffolds. Representative confocal microscopy images of PCL/PDA (A), PCL/PDA + GSNO (B), PCL/PDA + exosome (C), and PCL/PDA + GSNO + exosome (D) scaffolds. Exosomes were labeled with PKH67 exosome staining solution and incubated with either PCL/PDA or PCL/PDA + GSNO scaffolds. As a control, PCL/ PDA or PCL/PDA + GSNO scaffolds were incubated with PKH67 alone. Scale bars for low magnification images represent $50 \mu \mathrm{m}$ and for high magnification images, $10 \mu \mathrm{m}$.

exosome; Figures $3 \mathrm{C}$ and 4C, PCL/PDA + GSNO + exosome; Figures 3D and 4D). PCL/PDA and PCL/PDA + GSNO scaffolds without exosomes were co-cultured with RAW264.7 cells or hBMSCs to serve as controls (PCL/PDA; Figures $3 \mathrm{~A}$ and 4A, PCL/PDA + GSNO; Figures 3B and 4B). Following $4 \mathrm{~h}$ and $24 \mathrm{~h}$ of co-culture, cells were fixed and analyzed by 

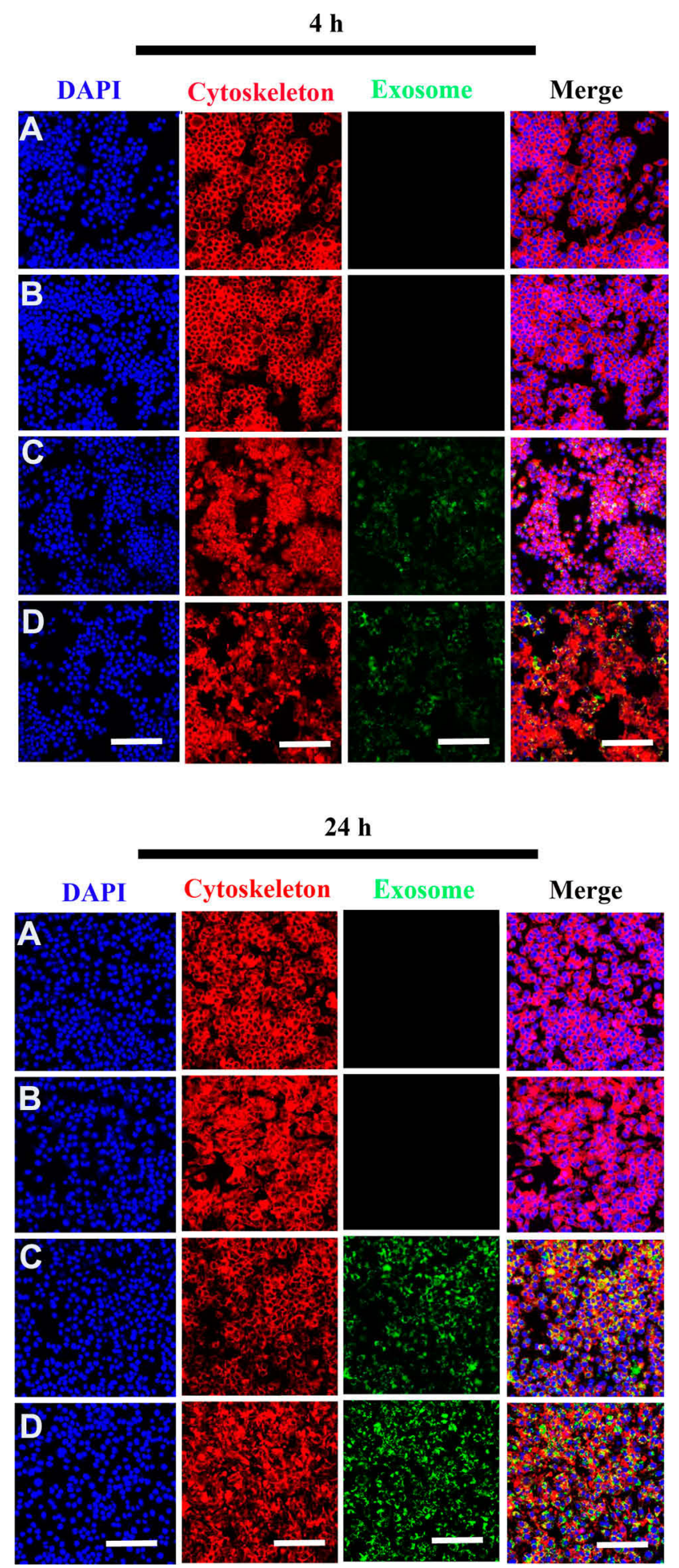

Figure 3 Internalization of scaffold-immobilized with exosomes by RAW 264.7 cells. Representative confocal microscopy images of RAW 264.7 cells co-cultured with PCL/PDA (A), PCL/PDA + GSNO (B), PCL/PDA + exosome (C), and PCL/ PDA + GSNO + exosome (D). Exosomes were pre-labeled with green fluorescent PKH67 prior to immobilization onto scaffolds. Cells were collected at 4 $\mathrm{h}$ and $24 \mathrm{~h}$ and stained with Alexa Fluor 594-conjugated phalloidin (red), and DAPI (blue). Scale bars: $100 \mu \mathrm{m}$. confocal microscopy. RAW 264.7 cells and hBMSCs cocultured with exosomes immobilized to PCL/PDA or PCL/ PDA + GSNO scaffolds demonstrated an increase in green fluorescent intensity compared with control (Figures 3C, 4C and 3D, 4D respectively). Furthermore, a similar pattern of exosome internalization by RAW264.7 cells or hBMSCs was observed between PCL/PDA and PCL/PDA + GSNO scaffolds.

\section{The Effect of Different Scaffolds on Inflammatory Response in Macrophages}

We next investigated the effect of scaffold composition on inflammatory macrophages. LPS-stimulated macrophages were co-cultured with scaffold-immobilized with exosomes and the morphology of the macrophages captured by confocal microscopy and SEM. The presence of either scaffold type resulted in a mixed population of inflammatory macrophages with two different morphologies. Similar cell spread areas were demonstrated between all scaffold conditions co-cultured with RAW 264.7 cells (Figure 5A and $\mathrm{B}$ ) however when exosomes were immobilized onto PCL/PDA + GSNO scaffolds, RAW 264.7 cells displayed a more elongated morphology compared with cells co-cultured with PCL/PDA alone (Figures 5A, $\mathrm{C}$ and $6 \mathrm{~A}$ ). To determine the impact of scaffold composition on inflammatory gene expression, RT-PCR was carried out. GSNO-modified and scaffold-immobilized with exosomes resulted in a significant downregulation of inflammatory gene expression compared with PCL/PDA control (Figure 6B). Notably, gene expression of IL-6, TNF- $\alpha$, iNOS, and IL- $1 \beta$ decreased by 5-, 2.5-, 1.6-, and 2.3-fold respectively for the PCL/PDA + GSNO + exosome group compared with PCL/PDA control.

\section{The Effect of Different Scaffolds on the Osteogenesis of hBMSCs}

To investigate the effect of scaffold composition on the osteogenic differentiation of hBMSCs, cells were co-cultured with PCL/PDA, PCL/PDA + GSNO, PCL/PDA + exosome and PCL/PDA + GSNO + exosome scaffolds and ALP protein expression assessed by immunofluorescence staining. ALP expression was greater when exosomes were immobilized onto PCL/PDA and PCL/PDA + GSNO scaffolds compared with PCL/PDA and PCL/PDA + GSNO scaffolds without exosomes (Figure 7A). To evaluate osteogenic differentiation 


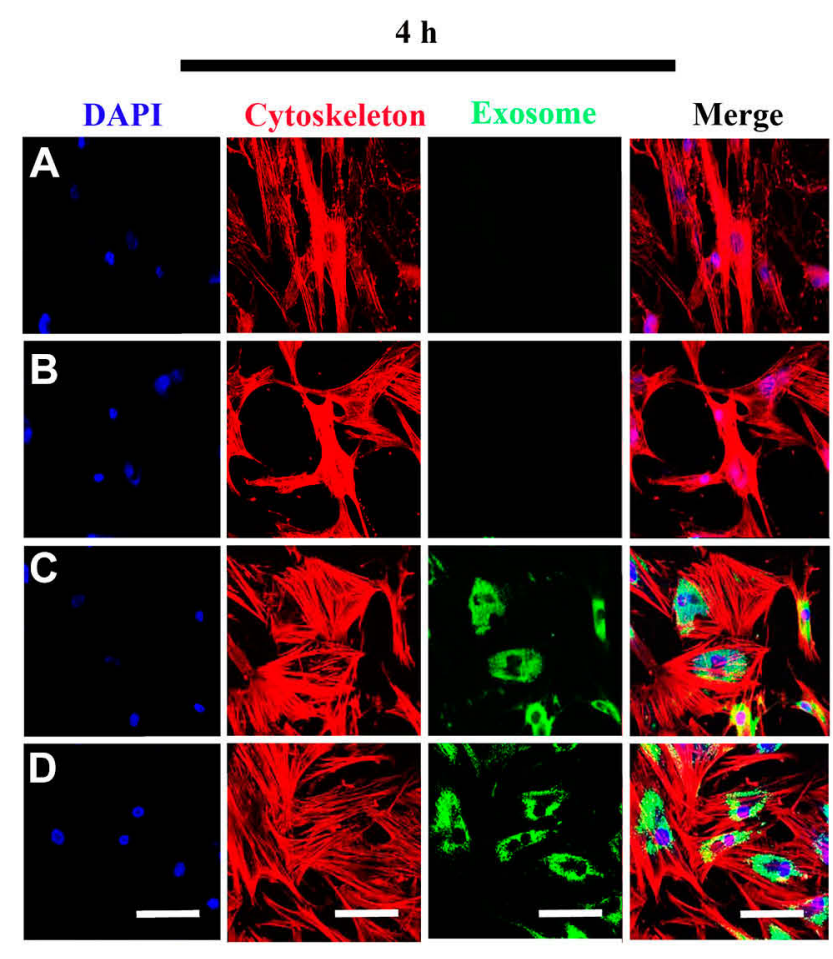

$24 \mathrm{~h}$

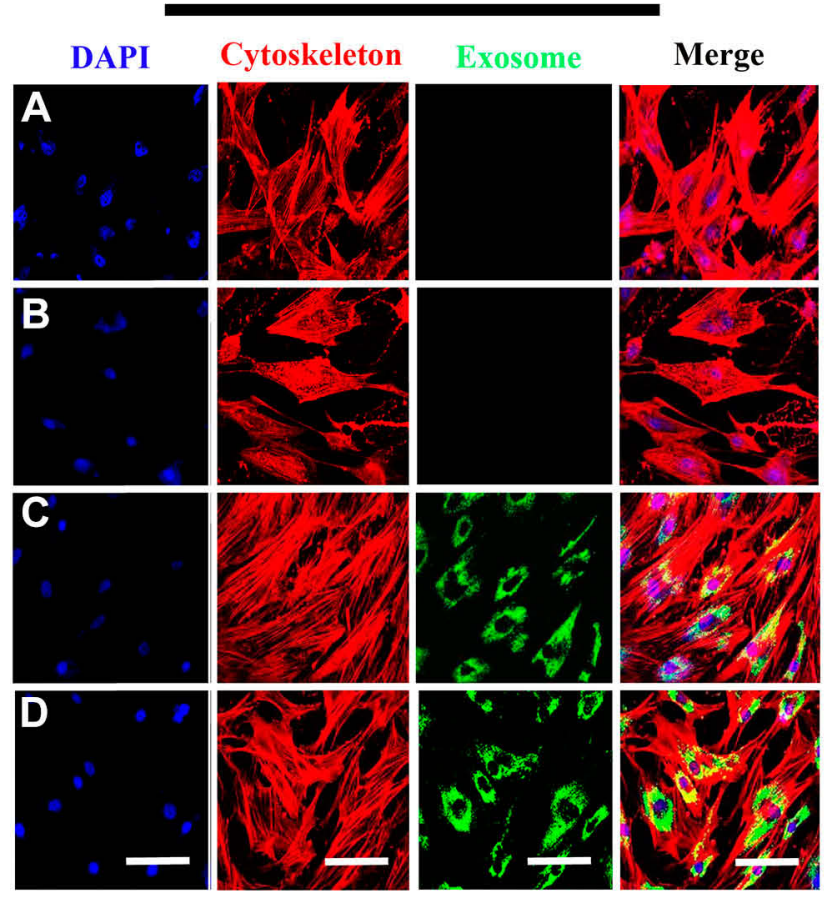

Figure 4 Internalization of scaffold-immobilized with exosomes by hBMSCs. Representative confocal microscopy images of hBMSCs co-cultured with PCL/PDA (A), PCL/PDA + GSNO (B), PCL/PDA + exosome (C), and PCL/PDA + GSNO + exosome (D). Exosomes were pre-labeled with green fluorescent $\mathrm{PKH} 67$ prior to immobilization onto scaffolds. Cells were collected at $4 \mathrm{~h}$ and $24 \mathrm{~h}$ and stained by Alexa Fluor 594-conjugated phalloidin (red), and DAPI (blue). Scale bars: $100 \mu \mathrm{m}$.

further, gene expression of osteogenic differentiation markers (ALP, Col-I, Runx2, and BMP-2) were assessed by RT-PCR (Figure 7B). mRNA expressions of ALP, Col-I, and Runx2 was significantly higher in exosomes immobilized onto PCL/ PDA and PCL/PDA + GNSO scaffolds compared to their respective scaffold groups without exosomes. We further evaluated ALP function by assessing protein activity using an ALP activity assay. ALP activity in hBMSCs was significantly increased when exosomes were immobilized onto PCL/ PDA and PCL/PDA + GNSO scaffolds (Figure 7C). Collectively, these results suggest PCL/PDA + exosome and PCL/PDA + GSNO + exosome scaffolds modulate differentiation of hBMSCs compared with their respective scaffolds types without exosomes, suggesting incorporating exosomes onto PCL/PDA bio-membrane scaffolds have profound proosteogenic effects.

\section{Discussion}

From the perspective of clinical management, the treatment of fracture, and/or post-traumatic complications resulting from large bone defects remains unsatisfactory. Current therapeutic methods to accelerate bone regeneration involves bone grafting, stem cell-based immune therapy, and bone graft materials/cytokines-related methods. ${ }^{26}$ To accelerate tissue regeneration and minimize side-effects associated with biomaterials, bioabsorbable membranes have gained increasing attention due to the single-step procedure, diverse prefabrication and modification potential. ${ }^{27}$ Theoretically, the local application of bioabsorbable membranes at the fracture site may regulate factors which mediate early bone forming environment which in turn could impact bone regeneration. As a biodegradable and bioresorbable polymer, PCL has been extensively used in various tissue regeneration and drug delivery fields. As PCL is inherently hydrophobic and has low wettability this has resulted in a huge knowledge gap in the modification of PCL scaffolds to accelerate bone regeneration. As a consequence, the purpose of this study was to develop a modified PCL scaffold with the capacity to regulate the bone forming environment and osteogenesis.

The bone healing process is complex and involves three consecutive and overlapping stages: hematoma formation, callus formation, and bone remodeling. Although previous data suggests that a certain degree of inflammation is essential for bone regeneration, a persistent or unregulated inflammatory stage is detrimental to bone regeneration. ${ }^{28}$ For example, as evidenced in our previous study the proinflammatory cytokine interleukin 1 beta was extensively expressed in the large delayed healing defects. ${ }^{20}$ Therefore, in this study we describe the simple modification of a PCL scaffold to regulate inflammation and enhance osteogenesis. 
A

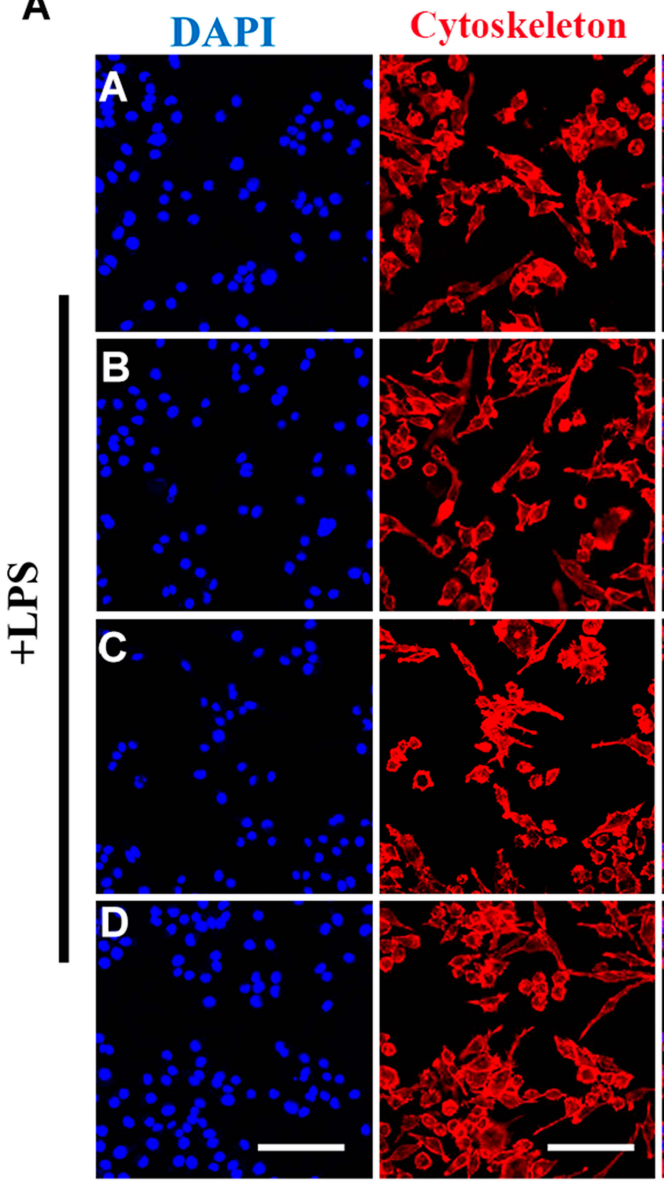

B

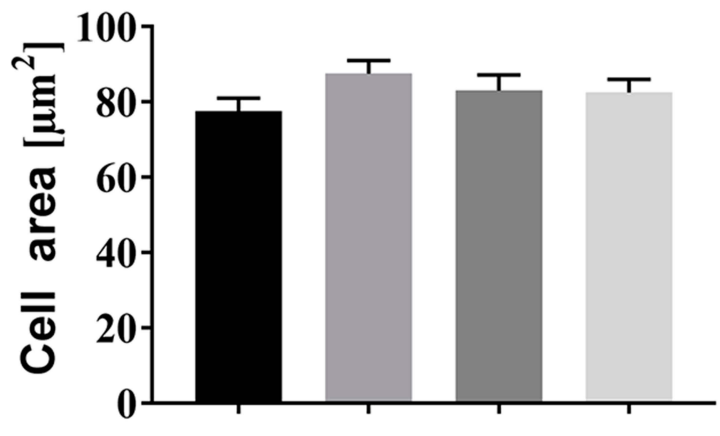

PCL/PDA

GSNO

\section{Exosome}

Cell spreading

$+\quad+\quad+$

$\begin{array}{lll}- & + & -\end{array}$

$+\quad+$
Merge
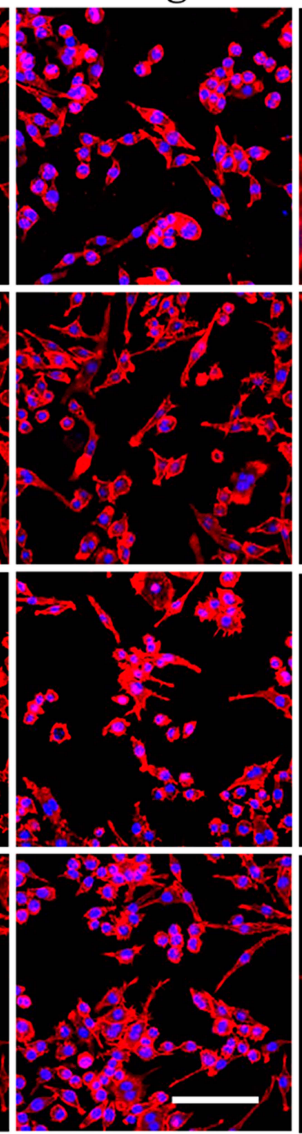

C

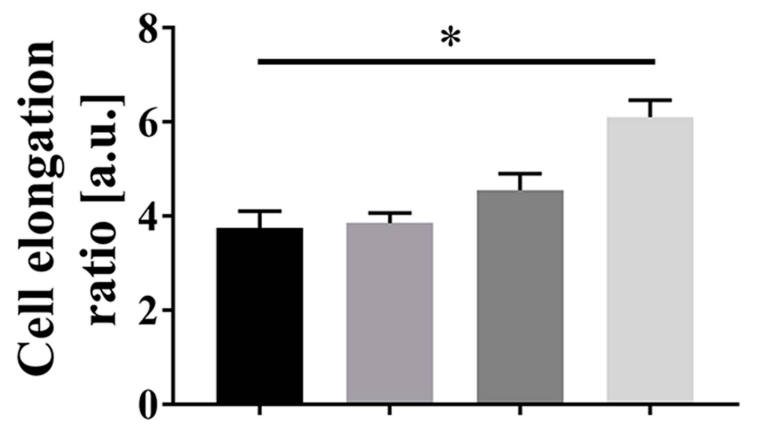

$+\quad+\quad+\quad+$

- $+\quad-\quad+$

$\begin{array}{llll}- & - & + & +\end{array}$

Figure 5 Scaffold-induced changes in macrophage morphology captured by confocal microscopy after lipopolysaccharide (LPS) stimulation. (A) Representative confocal microscopy images of RAW264.7 cells co-cultured with PCL/PDA (a), PCL/PDA + GSNO (b), PCL/PDA + exosome (c), and PCL/PDA + GSNO + exosome (d) scaffolds.

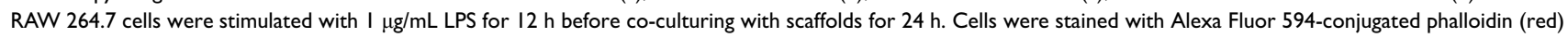
and DAPI (blue). Scale bars: $100 \mu \mathrm{m}$ for DAPI, Cytoskeleton, and Merge panels. In high magnification panel, scale bars represent $10 \mu \mathrm{m}$ (left) and $50 \mu \mathrm{m}$ (right). (B-C) Quantification of cell area ratio $(\mathbf{B})$ and elongation ratio $(\mathbf{C})$. ImageJ was used to quantify the macrophage area and elongation ratios. Error bars denote mean \pm SD of 50 individual cells. ${ }^{*} p<0.05$ relative to PCL/PDA group. 


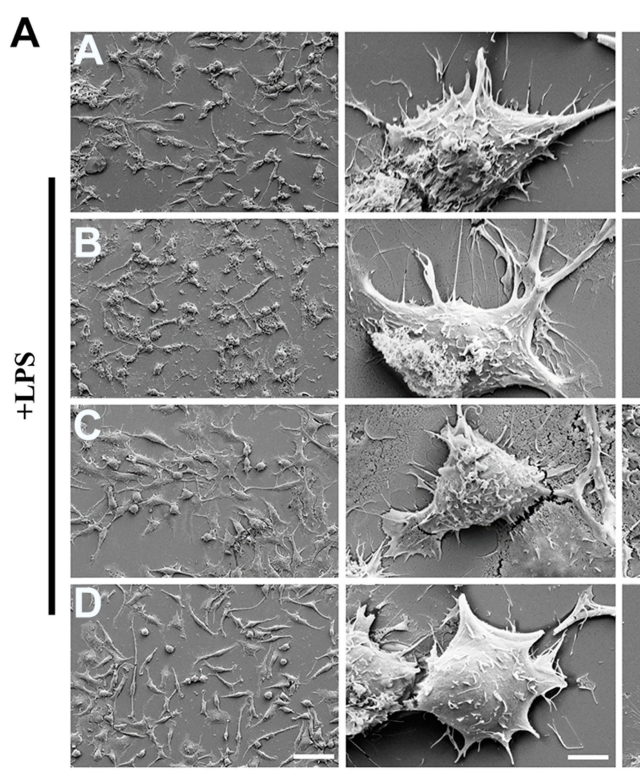

B
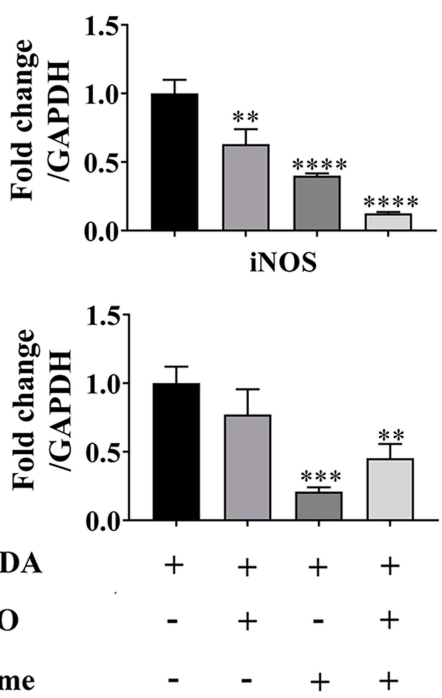

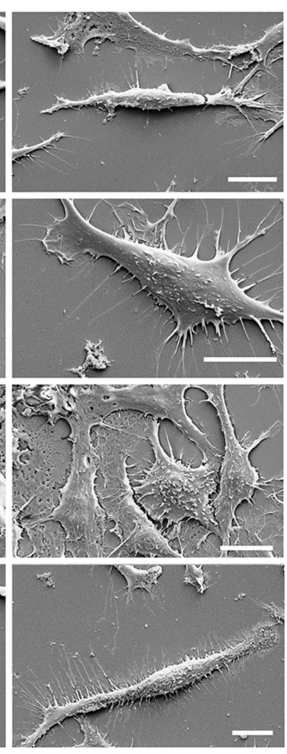

TNF- $\alpha$
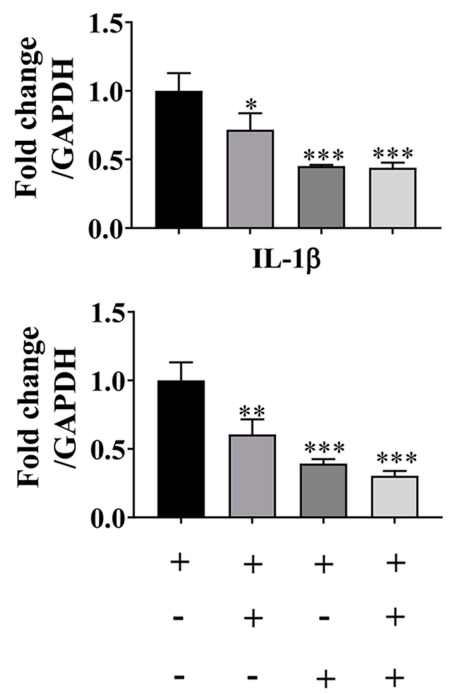

Figure 6 Scaffold-induced changes in macrophage morphology captured by scanning electron microscopy (SEM) after LPS stimulation. (A) Representative SEM images of RAW264.7 cells co-cultured with PCL/PDA (a), PCL/PDA + GSNO (b), PCL/PDA + exosome (c), and PCL/PDA + GSNO + exosome (d) scaffolds. RAW 264.7 cells were stimulated with I $\mu \mathrm{g} / \mathrm{mL}$ LPS for $12 \mathrm{~h}$ before co-culturing with scaffolds for $24 \mathrm{~h}$. Scale bars: $2 \mu \mathrm{m}$ (left panel), I $\mu \mathrm{m}$ (middle and right panel). (B) Relative expression of inflammatory genes in RAW 264.7 cells after I2h stimulation with LPS and co-culture with scaffolds for a further $24 \mathrm{~h}$. Values were normalized to the housekeeping gene, GAPDH. Error bars denote mean \pm SD. $*_{p}<0.05,{ }^{* *} p<0.01$, ***p $<0.0005$, ****p $<0.0001$ relative to PCL/PDA control.

Nitric oxide (NO) is a naturally generated bioactive molecule in mammals with a primary role as messenger molecule to regulate variety of physiological and pathophysiological functions, including maintaining cardiovascular homeostasis, central neuronal transmission, and immune response. ${ }^{29}$ As such, modulating NO levels has gained increasing interest as a therapeutic focus area. Over the last few decades, NOgenerating scaffolds that deliver exogenous $\mathrm{NO}$ or NO prodrugs have been developed via various physical and chemical techniques to accelerate tissue regeneration. ${ }^{30}$ The current paradigm is that $\mathrm{NO}$ is highly expressed during fracture healing and therefore an increase of NO levels via an exogeneous NOdonor could significantly impact fracture healing. ${ }^{29}$ S-nitrosothiols such as GSNO is one of the most widely used NO donors due to its long lasting NO release capacity. ${ }^{31}$ Several GSNO-mediated scaffolds have been previously examined in a number of studies. For example, GSNOincorporated pluronic F-127 (PL)-chitosan (CS) hydrogel was developed as a potential NO delivery scaffold. ${ }^{32}$ One of the pilot studies using GSNO-coated polymeric films also 
A

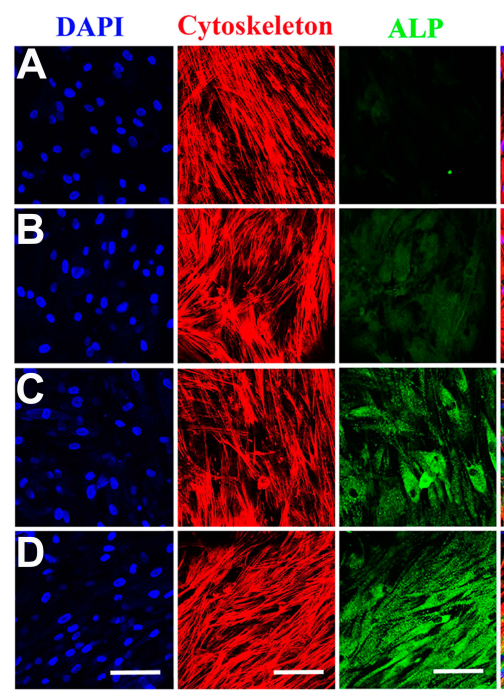

B

ALP

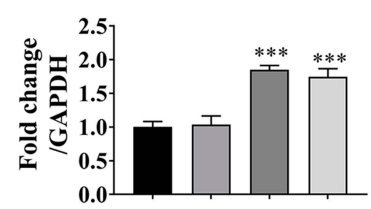

Runx2

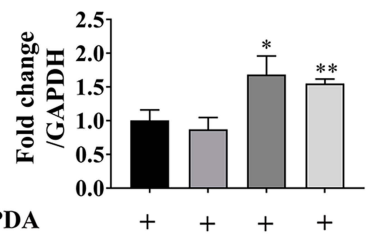

PCL/PDA

GSNO

Exosome
Merge

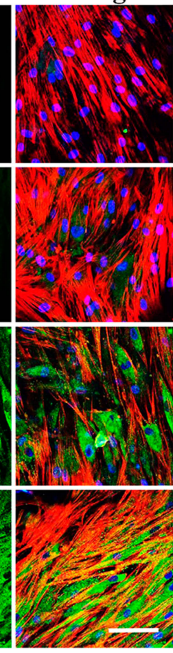

Col-I

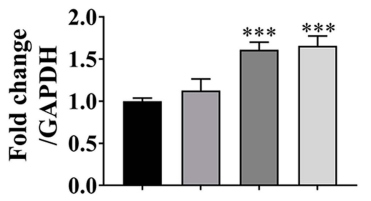

BMP-2

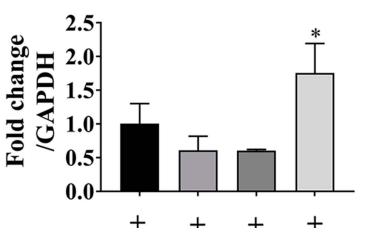

$++++$

$-+-+$

$-\quad++$

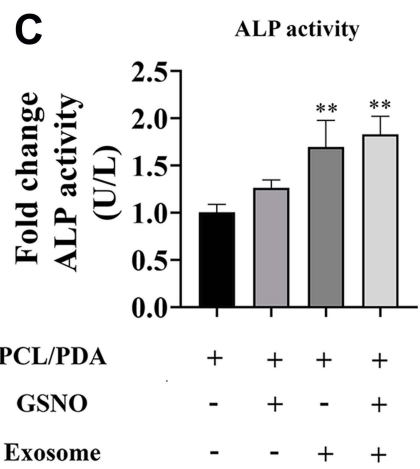

Figure 7 The effect of different scaffolds on the osteogenic differentiation of hBMSCs. (A) Representative confocal microscopy images of hBMSCs supplemented with osteogenic differentiation medium and co-cultured with PCL/PDA (a), PCL/PDA + GSNO (b), PCL/PDA + exosome (c), and PCL/PDA + GSNO + exosome (d) scaffolds. Cells were stained by Alexa Fluor 594-conjugated phalloidin (red), alkaline phosphatase (ALP; green), and DAPI (blue). All scale bars: 200 $\mu \mathrm{m}$. (B) Relative expression of osteogenic-related genes from samples treated as (A) and collected at $3 \mathrm{~d}$ post-seeding. Error bars denote mean \pm SD. $* p<0.05$, $* * p<0.01$, ${ }^{* *} p<0.0005$ relative to PCL/PDA control. (C) hBMSCs were cocultured with different scaffolds as (A) and ALP activity measured after $7 \mathrm{~d}$ in culture. Error bars denote the mean $\pm S D(n=3)$. ${ }^{*} p<0.01$ relative to PCL/ PDA control. demonstrated excellent mechanical and biological properties both in vitro and in vivo. ${ }^{33}$ In addition, GSNO-incorporated type I collagen nanofibrous scaffolds have demonstrated good fibroblast attachment and spreading properties compared with a silk fibroin-polyvinyl alcohol (SF-PVA) scaffold, indicating therapeutic potential for the treatment of ischemic non-healing ulcers. $^{34}$ Besides regulating cellular signaling pathways, GSNO may also act directly as an important regulator of inflammation although at present its regulatory mechanism is still unclear. ${ }^{35}$ For example, there is in vivo evidence that decreased levels of GSNO in the lungs of asthmatics as a result of increased levels of GSNO reductase (GSNOR), suggests that pharmacological suppression of GSNOR is able to significantly reduce inflammation. In addition, anti-neural and vascular inflammation properties of GSNO have also been reported in various stroke and traumatic brain injury models. $^{36-38}$ Administration of GSNO into focal cerebral ischemia rats resulted in infarction reduction and improved cerebral blood flow, followed by a significant reduction of inflammatory cytokine expression. ${ }^{36}$ Our data supports this notion and prompted us to further evaluate inflammatory cytokine expression levels in inflammatory macrophages after GSNO coating. In the present study, we performed assays to determine the effect of a GSNO-modified PCL scaffold on inflammation and detected differential expression of inflammatory cytokines between the GSNO-modified and the PCL/ PDA group. A striking feature of the GSNO-modified PCL scaffold was the significant reduction of inflammatory cytokine expression.

Mesenchymal stem cells (MSCs) are multi-potent cells with self-renewal and differentiation capacity, which represents one of the most commonly used tools for cell-based therapy and scaffold fabrication. ${ }^{39}$ For example, 3D-printed polyetherketoneketone scaffolds with human synovial fluid MSCs cultured on top showed superior bone regeneration compared with polyetherketoneketone control. ${ }^{40}$ In addition, a Phase I/II randomized clinical trial also suggested that a stem cell-based therapeutic method can accelerate craniofacial bone defects regeneration compared with the traditional guided bone regeneration therapy. ${ }^{41}$ However, long-term safety of MSCs-derived therapy and its potential tumorigenicity remains poorly explored. ${ }^{42}$ Exosomes are nano-sized, membrane-enclosed vesicles derived from endosomal origin, capable of transferring important macromolecules, such as proteins, lipids and nucleic acids (DNA, mRNA, microRNAs (miRNA) and long non-coding RNA 
(lncRNA)) from parental cells to recipient cells. ${ }^{43}$ Being non-living, non-replicative and cell free, exosome-mediated therapeutic methods represent a cheaper and safer treatment than conventional MSC-based methods. ${ }^{44}$ Exosomes derived from MSCs have the potential in becoming essential players in bone regeneration due to their important immune- and osteogenesis-regulatory potential. Many studies have demonstrated the therapeutic potential of MSCderived exosomes. For example, exosomes isolated from human adipose-derived MSCs can significantly promote angiogenesis in vitro and in vivo. ${ }^{45}$ The efficacy of MSCderived exosomes in the treatment of temporomandibular joint osteoarthritis (TMJ-OA) has also been investigated recently. ${ }^{46}$ The results indicated MSC-derived exosomes can significantly reduce inflammation and restore matrix expression in TMJ-OA animal models. ${ }^{46}$ Recent progress also demonstrated wide-ranging therapeutic potential of MSC-derived exosomes in liver injury, ${ }^{47}$ lung injury, ${ }^{48}$ traumatic brain injury. ${ }^{49}$

Therefore, in this study, we isolated exosomes from MSCs using a commercially available kit. Several different methods have been developed for exosome isolation over the past decade, which have mostly involved ultracentrifugation. ${ }^{50}$ For example, one study compared the results in exosome yield, purity and quality using four commercial kits and conventional ultracentrifugation. Each method produced different exosomes yields and size-distributions of which the kit from Invitrogen generated the largest exosome yield. ${ }^{50}$ In order to ensure a sufficient concentration of MSC-isolated exosomes in our study, all conditioned medium was subjected to isolation using the commercially available kit. It is well-known that several different criteria like TEM, NTA, flow cytometry, and exosome-specific markers (eg CD9, CD63, and CD81) are frequently used to characterize exosomes. In this study, we used TEM and NTA for characterization studies. Most reports reveal that exosomes exhibit a cup-shaped morphology with expression of exosome-specific markers. ${ }^{51}$ Using TEM analysis, we observed the presence of cup-shaped exosomes. However, in addition to TEM, other characterization methods, such as testing for exosome-specific markers are still needed for exosome characterization in the future. A previous study demonstrated MSC-derived exosomes range between $60 \mathrm{~nm}$ and $150 \mathrm{~nm}$ in size, ${ }^{52}$ while another study demonstrated $98 \mathrm{~nm}$ in diameter. ${ }^{53}$ By using NTA, we found the exosomes isolated in this study fell into two major size peaks; approximately 50 $\mathrm{nm}$ and approximately $120 \mathrm{~nm}$ in diameter. Exosome uptake is widely believed to be a multi-mechanism guided internalization process where particle size distributions can significantly affect the extent of exosome internalization. ${ }^{54}$ In the present study, exosome uptake analysis using the membrane marker, PKH67 and confocal microscopy, revealed internalization of exosomes by macrophages and hBMSCs demonstrated a similar exosome uptake pattern. Following $12 \mathrm{~h}$ of coculture with scaffold-immobilized exosomes, green fluorescence puncta accumulated inside cells.

We next investigated whether MSC-derived exosomes would regulate inflammation and promote tissue regeneration. Theoretically, MSC-derived exosomes can interact with different scaffolds, forming exosome layers with different releasing properties that are responsible for regulating cellular behavior. Previous studies have demonstrated MSC-derived exosome-modified scaffolds significantly enhanced osteogenesis and improved bone healing. ${ }^{55}$ For example, exosomes isolated from human-induced pluripotent stem cell-derived mesenchymal stem cells (hiPS-MSC-Exos) could significantly enhance osteogenesis and osteoinductivity of tricalcium phosphate $(\beta$-TCP) via the PI3K/Akt signaling pathway. ${ }^{55}$ A recent study also revealed MSC-derived extracellular vehicles (EVs) immobilized titanium (Ti) surfaces demonstrated enhanced MSC adhesion and proliferation after exosome immobilization ${ }^{56}$ revealing the regulatory potential of MSC-derived exosomes on cellular behavior. By conducting in vitro exosome immobilization onto PCL/PDA and PCL/PDA + GSNO scaffolds, we measured the internalization of MSC-derived exosomes into macrophages and hBMSCs. Ideally, different concentrations of exosomes should be applied to generate concentration-dependent scaffolds however, to the best of our knowledge, no specific and consistent concentration are known so far. ${ }^{57,58}$ We observed a significant reduction in the expression of inflammatory genes in the GSNO-modified PCL/PDA scaffold compared with PCL/PDA control. Upon immobilization with exosomes onto the scaffolds, inflammatory gene expression was further decreased suggesting there may be a synergistic effect on inflammatory gene expression in the GSNO and MSCderived exosomes group. It may also indicate that immobilized MSC-derived exosomes have a regulatory effect, possibly owing to microRNA cargos inside the exosomes. ${ }^{53}$

\section{Conclusion}

In summary, the present study demonstrates a novel way to modify conventional PCL surfaces. Constructing a PCL scaffold with immunoregulatory potential was developed by combining GSNO and MSCs-derived exosomes. We were able to confirm it could significantly reduce the inflammation stimulated by inflammatory macrophages and importantly, 
this modification further accelerated osteogenic differentiation of mesenchymal stem cells. The results obtained in this study provides us with a better understanding of GSNO- and exosome-based scaffold modifications and supports the hypothesis that this one-step modification technique may hold clinical translational promise for guiding osteogenesis and improving the repair and regeneration of skeletal tissues. However, further research is required to evaluate its biological activity with in vivo studies.

\section{Acknowledgments}

This work was financially supported by the National Natural Science Foundation of China (Grant No. 31960209), Guizhou Science and Technology Fund Project (Grant No. [2020]1Y093), the Scientific Innovation Foundation for Returned Overseas Chinese Scholars of Guizhou Province (Grant No. 2018-07), and Academic New Seedlings Cultivation and Innovation Exploration Project of Zunyi Medical University (Grant No. [2017]5733-015).

\section{Author Contributions}

All authors made substantial contributions to conception and design, acquisition of data, or analysis and interpretation of data; took part in drafting the article or revising it critically for important intellectual content; gave final approval of the version to be published; and agree to be accountable for all aspects of the work.

\section{Disclosure}

The authors declare that they have no conflict of interest.

\section{References}

1. Wang X, Friis T, Glatt V, Crawford R, Xiao Y. Structural properties of fracture haematoma: current status and future clinical implications. J Tissue Eng Regen Med. 2017;11(10):2864-2875. doi:10.1002/ term. 2190

2. Gomez-Barrena E, Rosset P, Lozano D, Stanovici J, Ermthaller C, Gerbhard F. Bone fracture healing: cell therapy in delayed unions and nonunions. Bone. 2015;70:93-101. doi:10.1016/j.bone.2014.07.033

3. Goulet JA, Senunas LE, DeSilva GL, Greenfield ML. Autogenous iliac crest bone graft. Complications and functional assessment. Clin Orthop Relat Res. 1997;339:76-81. doi:10.1097/00003086199706000-00011

4. Xu XY, Li X, Wang J, He XT, Sun HH, Chen FM. Concise review: periodontal tissue regeneration using stem cells: strategies and translational considerations. Stem Cells Transl Med. 2018.

5. Ma Y, Zhou Y, Wu F, Ji W, Zhang J, Wang X. The bidirectional interactions between inflammation and coagulation in fracture hematoma. Tissue Eng Part B Rev. 2019;25(1):46-54. doi:10.1089/ ten.teb.2018.0157

6. Galliot B, Crescenzi M, Jacinto A, Tajbakhsh S. Trends in tissue repair and regeneration. Development. 2017;144(3):357-364. doi:10. 1242/dev. 144279
7. Ciuffi S, Zonefrati R, Brandi ML. Adipose stem cells for bone tissue repair. Clin Cases Miner Bone Metab. 2017;14(2):217-226. doi:10.11138/ccmbm/2017.14.1.217

8. Yang Y, Rossi FMV, Putnins EE. Periodontal regeneration using engineered bone marrow mesenchymal stromal cells. Biomaterials. 2010;31(33):8574-8582. doi:10.1016/j.biomaterials.2010.06.026

9. Iwata T, Washio K, Yoshida T, et al. Cell sheet engineering and its application for periodontal regeneration. J Tissue Eng Regen Med. 2015;9(4):343-356. doi:10.1002/term.1785

10. Mazini L, Rochette L, Amine M, Malka G. Regenerative capacity of adipose derived stem cells (ADSCs), comparison with mesenchymal stem cells (MSCs). Int J Mol Sci. 2019;20(10):2523. doi:10.3390/ ijms 20102523

11. Sarko DK, McKinney CE. Exosomes: origins and therapeutic potential for neurodegenerative disease. Front Neurosci. 2017;11:82. doi:10.3389/fnins.2017.00082

12. Wortzel I, Dror S, Kenific CM, Lyden D. Exosome-mediated metastasis: communication from a distance. Dev Cell. 2019;49(3):347-360. doi:10.1016/j.devcel.2019.04.011

13. Teng X, Chen L, Chen W, Yang J, Yang Z, Shen Z. Mesenchymal stem cell-derived exosomes improve the microenvironment of infarcted myocardium contributing to angiogenesis and anti-inflammation. Cell Physiol Biochem. 2015;37(6):2415-2424. doi:10.1159/000438594

14. Lou GH, Chen Z, Zheng M, Liu YN. Mesenchymal stem cell-derived exosomes as a new therapeutic strategy for liver diseases. Exp Mol Med. 2017;49(6):e346-e346. doi:10.1038/emm.2017.63

15. Aghajani Nargesi A, Lerman LO, Eirin A. Mesenchymal stem cell-derived extracellular vesicles for kidney repair: current status and looming challenges. Stem Cell Res Ther. 2017;8(1):273. doi:10.1186/s13287-017-0727-7

16. Lee JH, Park J, Lee JW. Therapeutic use of mesenchymal stem cell-derived extracellular vesicles in acute lung injury. Transfusion. 2019;59(S1):876-883. doi:10.1111/trf.14838

17. De Jong OG, Van Balkom BW, Schiffelers RM, Bouten CV, Verhaar MC. Extracellular vesicles: potential roles in regenerative medicine. Front Immunol. 2014;5:608. doi:10.3389/fimmu.2014. 00608

18. Bateman RM, Ellis CG, Suematsu M, Walley KR. S-nitrosoglutathione acts as a small molecule modulator of human fibrin clot architecture. PLoS One. 2012;7(8):e43660. doi:10.1371/ journal.pone. 0043660

19. de Menezes AM, de Souza GF, Gomes AS, et al. S-nitrosoglutathione decreases inflammation and bone resorption in experimental periodontitis in rats. J Periodontol. 2012;83(4):514-521. doi:10.1902/ jop.2011.110332

20. Wang X, Friis TE, Masci PP, Crawford RW, Liao W, Xiao Y. Alteration of blood clot structures by interleukin-1 beta in association with bone defects healing. Sci Rep. 2016;6:35645. doi:10.1038/ srep35645

21. Lima B, Lam GK, Xie L, et al. Endogenous S-nitrosothiols protect against myocardial injury. Proc Natl Acad Sci U S A. 2009;106 (15):6297-6302. doi:10.1073/pnas.0901043106

22. Shahrezaee M, Salehi M, Keshtkari S, Oryan A, Kamali A, Shekarchi B. In vitro and in vivo investigation of PLA/PCL scaffold coated with metformin-loaded gelatin nanocarriers in regeneration of critical-sized bone defects. Nanomedicine. 2018;14(7):2061-2073. doi:10.1016/j.nano.2018.06.007

23. Kamath MS, Ahmed SS, Dhanasekaran M, Santosh SW. Polycaprolactone scaffold engineered for sustained release of resveratrol: therapeutic enhancement in bone tissue engineering. Int J Nanomedicine. 2014;9:183-195. doi:10.2147/IJN.S49460

24. Schmittgen TD, Livak KJ. Analyzing real-time PCR data by the comparative C(T) method. Nat Protoc. 2008;3(6):1101-1108. doi:10.1038/nprot.2008.73 
25. Wei F, Li Z, Crawford R, Xiao Y, Zhou Y. Immunoregulatory role of exosomes derived from differentiating mesenchymal stromal cells on inflammation and osteogenesis. J Tissue Eng Regen Med. 2019;13 (11):1978-1991. doi:10.1002/term.2947

26. Jimi E, Hirata S, Osawa K, Terashita M, Kitamura C, Fukushima H. The current and future therapies of bone regeneration to repair bone defects. Int J Dent. 2012;2012:148261. doi:10.1155/2012/148261

27. Wang J, Wang L, Zhou Z, et al. Biodegradable polymer membranes applied in guided bone/tissue regeneration: a review. Polymers (Basel). 2016;8(4):115.

28. Wendler S, Schlundt $\mathrm{C}$, Bucher $\mathrm{CH}$, et al. Immune modulation to enhance bone healing-a new concept to induce bone using prostacyclin to locally modulate immunity. Front Immunol. 2019;10:713. doi:10.3389/fimmu.2019.00713

29. Diwan AD, Wang MX, Jang D, Zhu W, Murrell GA. Nitric oxide modulates fracture healing. J Bone Miner Res. 2000;15(2):342-351. doi:10.1359/jbmr.2000.15.2.342

30. Zhou X, Zhang J, Feng G, Shen J, Kong D, Zhao Q. Nitric oxide-releasing biomaterials for biomedical applications. Curr Med Chem. 2016;23(24):2579-2601. doi:10.2174/092986732366616072 9104647

31. Liang H, Nacharaju P, Friedman A, Friedman JM. Nitric oxide generating/releasing materials. Future Sci OA. 2015;1(1). doi: $10.4155 /$ fso. 15.54

32. Pelegrino MT, De Araujo Lima B, Do Nascimento MHM, Lombello CB, Brocchi M, Seabra AB. Biocompatible and antibacterial nitric oxide-releasing pluronic F-127/chitosan hydrogel for topical applications. Polymers (Basel). 2018;10(4):452. doi:10.3390/ polym 10040452

33. Yoo JW, Acharya G, Lee CH. In vivo evaluation of vaginal films for mucosal delivery of nitric oxide. Biomaterials. 2009;30(23-24):3978-3985. doi:10.1016/j.biomaterials.2009.04.004

34. Ramadass SK, Nazir LS, Thangam R, et al. Type I collagen peptides and nitric oxide releasing electrospun silk fibroin scaffold: a multifunctional approach for the treatment of ischemic chronic wounds. Colloids Surf B Biointerfaces. 2019;175:636-643. doi:10.1016/j.colsurfb.2018.12.025

35. Won JS, Kim J, Annamalai B, Shunmugavel A, Singh I, Singh AK. Protective role of S-nitrosoglutathione (GSNO) against cognitive impairment in rat model of chronic cerebral hypoperfusion. J Alzheimers Dis. 2013;34(3):621-635. doi:10.3233/JAD-121786

36. Khan M, Sekhon B, Giri S, et al. S-Nitrosoglutathione reduces inflammation and protects brain against focal cerebral ischemia in a rat model of experimental stroke. J Cereb Blood Flow Metab. 2005;25(2):177-192. doi:10.1038/sj.jcbfm.9600012

37. Khan M, Sakakima H, Dhammu TS, et al. S-nitrosoglutathione reduces oxidative injury and promotes mechanisms of neurorepair following traumatic brain injury in rats. $J$ Neuroinflammation. 2011;8:78. doi:10.1186/1742-2094-8-78

38. Khan M, Im YB, Shunmugavel A, et al. Administration of S-nitrosoglutathione after traumatic brain injury protects the neurovascular unit and reduces secondary injury in a rat model of controlled cortical impact. J Neuroinflammation. 2009;6:32. doi:10.1186/ 1742-2094-6-32

39. Yamagata K, Nakayamada S, Tanaka Y. Use of mesenchymal stem cells seeded on the scaffold in articular cartilage repair. Inflamm Regen. 2018;38:4. doi:10.1186/s41232-018-0061-1

40. Lin Y, Umebayashi M, Abdallah MN, et al. Combination of polyetherketoneketone scaffold and human mesenchymal stem cells from temporomandibular joint synovial fluid enhances bone regeneration. Sci Rep. 2019;9(1):472. doi:10.1038/s41598-018-36778-2
41. Kaigler D, Pagni G, Park CH, et al. Stem cell therapy for craniofacial bone regeneration: a randomized, controlled feasibility trial. Cell Transplant. 2013;22(5):767-777. doi:10.3727/096368912X652968

42. Squillaro T, Peluso G, Galderisi U. Clinical trials with mesenchymal stem cells: an update. Cell Transplant. 2016;25(5):829-848. doi:10.3727/096368915X689622

43. Wang J, Sun X, Zhao J, et al. Exosomes: a novel strategy for treatment and prevention of diseases. Front Pharmacol. 2017;8:300. doi:10.3389/fphar.2017.00300

44. Willis GR, Kourembanas S, Mitsialis SA. Toward exosome-based therapeutics: isolation, heterogeneity, and fit-for-purpose potency. Front Cardiovasc Med. 2017;4:63. doi:10.3389/fcvm.2017.00063

45. Liang X, Zhang L, Wang S, Han Q, Zhao RC. Exosomes secreted by mesenchymal stem cells promote endothelial cell angiogenesis by transferring miR-125a. J Cell Sci. 2016;129(11):2182-2189. doi: $10.1242 /$ jes. 170373

46. Zhang S, Teo KYW, Chuah SJ, Lai RC, Lim SK, Toh WS. MSC exosomes alleviate temporomandibular joint osteoarthritis by attenuating inflammation and restoring matrix homeostasis. Biomaterials. 2019;200:35-47. doi:10.1016/j.biomaterials.2019.02.006

47. Damania A, Jaiman D, Teotia AK, Kumar A. Mesenchymal stromal cell-derived exosome-rich fractionated secretome confers a hepatoprotective effect in liver injury. Stem Cell Res Ther. 2018;9 (1):31. doi:10.1186/s13287-017-0752-6

48. Xu N, Shao Y, Ye K, et al. Mesenchymal stem cell-derived exosomes attenuate phosgene-induced acute lung injury in rats. Inhal Toxicol. 2019;31(2):52-60. doi:10.1080/08958378.2019.1597220

49. Ni H, Yang S, Siaw-Debrah F, et al. Exosomes derived from bone mesenchymal stem cells ameliorate early inflammatory responses following traumatic brain injury. Front Neurosci. 2019;13:14. doi:10.3389/fnins.2019.00014

50. Patel GK, Khan MA, Zubair H, et al. Comparative analysis of exosome isolation methods using culture supernatant for optimum yield, purity and downstream applications. Sci Rep. 2019;9(1):5335. doi:10.1038/s41598-019-41800-2

51. Lobb RJ, Becker M, Wen SW, et al. Optimized exosome isolation protocol for cell culture supernatant and human plasma. J Extracell Vesicles. 2015;4:27031. doi:10.3402/jev.v4.27031

52. Di Trapani M, Bassi G, Midolo M, et al. Differential and transferable modulatory effects of mesenchymal stromal cell-derived extracellular vesicles on T, B and NK cell functions. Sci Rep. 2016;6:24120. doi:10.1038/srep24120

53. Ferguson SW, Wang J, Lee CJ, et al. The microRNA regulatory landscape of MSC-derived exosomes: a systems view. Sci Rep. 2018;8(1):1419. doi:10.1038/s41598-018-19581-x

54. Caponnetto F, Manini I, Skrap M, et al. Size-dependent cellular uptake of exosomes. Nanomedicine. 2017;13(3):1011-1020. doi:10.1016/j.nano.2016.12.009

55. Zhang J, Liu X, Li H, et al. Exosomes/tricalcium phosphate combination scaffolds can enhance bone regeneration by activating the PI3K/Akt signaling pathway. Stem Cell Res Ther. 2016;7(1):136. doi:10.1186/s13287-016-0391-3

56. Wang X, Shah FA, Vazirisani F, et al. Exosomes influence the behavior of human mesenchymal stem cells on titanium surfaces. Biomaterials. 2020;230:119571. doi:10.1016/j.biomaterials.2019.119571

57. Wei F, Li M, Crawford R, Zhou Y, Xiao Y. Exosome-integrated titanium oxide nanotubes for targeted bone regeneration. Acta Biomater. 2019;86:480-492. doi:10.1016/j.actbio.2019.01.006

58. Takeuchi R, Katagiri W, Endo S, Kobayashi T. Exosomes from conditioned media of bone marrow-derived mesenchymal stem cells promote bone regeneration by enhancing angiogenesis. PLoS One. 2019;14(11):e0225472. doi:10.1371/journal.pone.0225472 


\section{Publish your work in this journal}

The International Journal of Nanomedicine is an international, peerreviewed journal focusing on the application of nanotechnology in diagnostics, therapeutics, and drug delivery systems throughout the biomedical field. This journal is indexed on PubMed Central, MedLine, CAS, SciSearch ${ }^{\circledR}$, Current Contents ${ }^{\circledR} /$ Clinical Medicine,
Journal Citation Reports/Science Edition, EMBase, Scopus and the Elsevier Bibliographic databases. The manuscript management system is completely online and includes a very quick and fair peer-review system, which is all easy to use. Visit http://www.dovepress.com/ testimonials.php to read real quotes from published authors. 